\title{
Intermediate Mass-Ratio Black Hole Binaries: Applicability of Small Mass-Ratio Perturbation Theory
}

\author{
Maarten van de Meent $\odot^{*}$ and Harald P. Pfeiffer \\ Max Planck Institute for Gravitational Physics (Albert Einstein Institute), Am Mühlenberg 1, D-14476 Potsdam, Germany
}

(Received 30 June 2020; accepted 14 September 2020; published 28 October 2020)

\begin{abstract}
The inspiral phasing of binary black holes at intermediate mass ratios $\left(m_{2} / m_{1} \sim 10^{-3}\right)$ is important for gravitational wave observations, but not accessible to standard modeling techniques: The accuracy of the small mass-ratio (SMR) expansion is unknown at intermediate mass ratios, whereas numerical relativity simulations cannot reach this regime. This article assesses the accuracy of the SMR expansion by extracting the first three terms of the SMR expansion from numerical relativity data for nonspinning, quasicircular binaries. We recover the leading term predicted by SMR theory and obtain a robust prediction of the nextto-leading term. The influence of higher-order terms is bounded to be small, indicating that the SMR series truncated at next-to-leading order is quite accurate at intermediate mass ratios and even at nearly comparable mass binaries. We estimate the range of applicability for SMR and post-Newtonian series for nonspinning, quasicircular inspirals.
\end{abstract}

DOI: $10.1103 /$ PhysRevLett.125.181101

Inspiraling and merging black hole $(\mathrm{BH})$ binaries are the most numerous source of gravitational waves (GWs) observed by the Laser Interferometer Gravitational Wave Observatory (LIGO) and Virgo detectors [1,2] and are one of the key science targets for third-generation ground-based GW detectors [3], as well as the space-based Laser Interferometer Space Antenna (LISA) observatory [4]. The mass ratio $q \equiv m_{2} / m_{1} \leq 1$ is one of the key parameters in the dynamics of these systems. The LIGO and Virgo observations [5-7] mostly report $q$ close to unity, with GW190412 [8] and GW190814 [9] the first systems with clearly unequal masses $(q \sim 0.28$ and $q \sim 0.11)$.

In the future, observations of binaries with lower $q$ are expected: Continued observations with the current detectors [10] may reveal binaries with smaller $q$. Third-generation ground-based detectors with improved low frequency sensitivity will be able to detect the capture of stellar mass BHs by intermediate mass $\mathrm{BHs}$ with mass ratios down to $q \sim 10^{-3}$ [11]. LISA will observe the mergers of massive BHs of millions of solar masses. While the majority of these are expected to have $q \gtrsim 0.1$, there could a significant tail of events down to $q \sim 0.01[12,13]$. LISA will also be sensitive to mergers of intermediate mass BHs with massive $\mathrm{BHs}\left(q \sim 10^{-3}\right)$ and extreme mass-ratio

Published by the American Physical Society under the terms of the Creative Commons Attribution 4.0 International license. Further distribution of this work must maintain attribution to the author(s) and the published article's title, journal citation, and DOI. Open access publication funded by the Max Planck Society. inspirals $\left(q \sim 10^{-5}\right)$ as sensitive probes of black hole physics [4].

The modeling of inspiraling binaries at all mass ratios is therefore of paramount importance for detection and analysis of GW sources. The three primary modeling approaches are post-Newtonian slow-velocity perturbation theory [14], numerical relativity (NR), i.e., direct numerical integration of the full nonlinear Einstein equations [15], and small massratio (SMR) perturbation theory [16]. Effective one-body methods [17] provide a means to combine and resum information from all three approaches and also from newer developments like post-Minkowski expansions [18].

This article examines whether the SMR and NR approaches combined can accurately model binaries with any mass ratio or whether there is a "gap" at intermediate mass ratios where neither SMR nor NR is sufficiently accurate. The SMR approximation expands the dynamics of a coalescing binary in powers of $q$ or the symmetric mass ratio $\nu \equiv m_{1} m_{2} /\left(m_{1}+m_{2}\right)^{2}=q+\mathcal{O}\left(q^{2}\right)$. At leading order, the secondary object follows a geodesic in the background space-time generated by the primary. The impact of the secondary's mass on the dynamics can be included as an effective force term, the gravitational selfforce (GSF). Calculation of the GSF has progressed rapidly over the past two decades (see [19] for a review), but the full next-to-leading-order contribution to the orbital phasing has not yet been obtained. While the main motivation for SMR lies in extreme mass-ratio inspirals, there is increasing evidence [20-26] that the SMR may be applicable even at comparable masses.

Numerical relativity directly solves the full nonlinear Einstein equations [15]. The vast majority of simulations 
performed to date are at comparable masses, with only very few simulations at $q \lesssim 0.1$ (see, e.g., [27], but note [28,29] for simulations from $q=1 / 18$ to $q=1 / 128$ ). The limited coverage in $q$ has two causes. First, the number of orbits the binary spends in the strong field region grows $\alpha \nu^{-1}$. Second, because of the Courant limit on the time step of the numerical simulations, the number of time steps per orbit increases $\propto q^{-1}$. Combined, these effects cause an increase in computational cost at least quadratically in mass ratio. The need for higher numerical resolution to resolve the ever smaller secondary (as $q \rightarrow 0$ ) and to preserve phase accuracy over the increasingly longer inspiral will increase computational cost further.

Given the expectation of binaries at all mass ratios, the question arises how to model intermediate mass-ratio binaries at small separation: post-Newtonian theory is not accurate close to merger, owing to the high velocities; numerical relativity simulations are limited to large mass ratios, $q \gtrsim 0.1$; and the SMR approximation is presently only available at leading order in $q$, and thus may be inaccurate at intermediate mass ratios. This Letter investigates the existence of a mass-ratio gap where none of the modeling approaches is applicable. We analyze NR simulations at mass ratios $0.1 \leq q \leq 1$ computed with the SpEC code $[27,30]$ and extract the first three terms in the SMR expansion of the orbital phasing. Analyzing these terms, we conclude that SMR results at next-to-leading order can likely bridge the mass-ratio gap up to mass ratios $q$ large enough to be covered by numerical relativity.

Methodology.-We use geometric units such that $c=G=1$ and examine the orbital phase extracted from the gravitational radiation at future null infinity,

$$
\phi \equiv \frac{1}{2} \arg h_{22} .
$$

Here $h_{22}$ is the spin weight $s=-2$ spherical harmonic $(\ell, m)=(2,2)$ mode of the complex GW strain. The current Letter focuses on nonprecessing binaries where Eq. (1) is sufficient.

Introducing the orbital frequency

$$
\Omega \equiv \frac{d \phi}{d t},
$$

we consider the orbital phase as a function of the orbital frequency $\phi(\Omega)$. In the SMR approximation, $\phi$ can be calculated by a two timescale expansion [31] leading to a power series in the mass ratio, known as the postadiabatic (PA) expansion,

$$
\phi(\Omega)=\sum_{n=0}^{\infty} \nu^{n-1} \phi_{n \mathrm{PA}}(M \Omega) .
$$

Here, $\phi_{n \mathrm{PA}}$ are functions of $M \Omega$, where $M \equiv m_{1}+m_{2}$ is the total mass of the binary. Alternatively, one can consider $\phi_{n \mathrm{PA}}$ as functions of $m_{1} \Omega$ and/or expand in $q$ as the small parameter (shown later).

The leading-order term [31,32] $\phi_{\mathrm{OPA}}$ (called "adiabatic" or "0 postadiabatic") is independent of the choice of expansion parameter or mass normalization. It can be computed by energy balance,

$$
\frac{d \phi_{0 \mathrm{PA}}}{d \Omega}=\nu \Omega \frac{d E}{d \Omega}\left(\frac{d E}{d t}\right)^{-1},
$$

where $E(\Omega)$ is the specific energy of the circular geodesic with orbital frequency $\Omega$, and $d E / d t$ is its energy loss to GWs. We compute $d E / d t$ with the Black Hole Perturbation Toolkit [33], utilizing the arbitrary precision Teukolsky code developed in [34-40], and denote the result as $\phi_{\text {OPA }}^{\mathrm{SMR}}$ below.

The 1PA term in the expansion requires knowledge of the full first-order GSF for nearly circular orbits, and the dissipative part of second-order GSF for quasicircular inspirals [31,32]. Calculation of the first-order GSF for nonspinning binaries is now routine [41-45]. The calculation of second-order GSF for quasicircular orbits, however, remains an open challenge in GSF theory, although steady progress has been made [46-53].

We use numerical relativity simulations from the SpEC code, which utilizes the quasilocal angular momentum formalism to monitor the black hole spins [54-57], iterative eccentricity reduction to achieve orbital eccentricities $e \lesssim 10^{-4}$ [58,59], and solves the Einstein evolution equations in the generalized harmonic formulation [60-63] with constraint damping and minimally reflective outer boundary conditions [63-65] (see [27] for more details). Because of the use of spectral methods and a dual-frame approach [66], SpEC achieves very high accuracies even for long inspiral simulations that cover a comparatively large range in orbital frequencies. Gravitational radiation is extracted using the Regge-Wheeler-Zerilli formalism, extrapolated to future null infinity $[27,67]$ and corrected for center of mass drifts [68].

This study utilizes $55 \mathrm{NR}$ simulations of nonspinning quasicircular inspirals from the public SXS catalog $[27,69]$ with mass ratios $q \in[0.1,1]$. The initial orbital frequency is in the range $M \Omega \sim 0.015, \ldots, 0.02$. Simulations with smaller $q$ tend to start at the higher frequencies, to achieve a computationally manageable overall duration of the simulations. All simulations are available at multiple numerical resolutions for convergence tests.

The orbital phase $\phi^{\mathrm{NR}}(M \Omega)$ is determined by locally fitting a low-order polynomial in $t$ to $\phi^{\mathrm{NR}}(t)$. The width of the fitting window is variable such that at low frequencies it encompasses several radial oscillations of any residual eccentricity in the simulations, while at larger frequencies it is small enough to avoid systematic bias due to the rapidly changing frequency. The constant of integration when integrating Eq. (2) is chosen such that $\phi=0$ at 


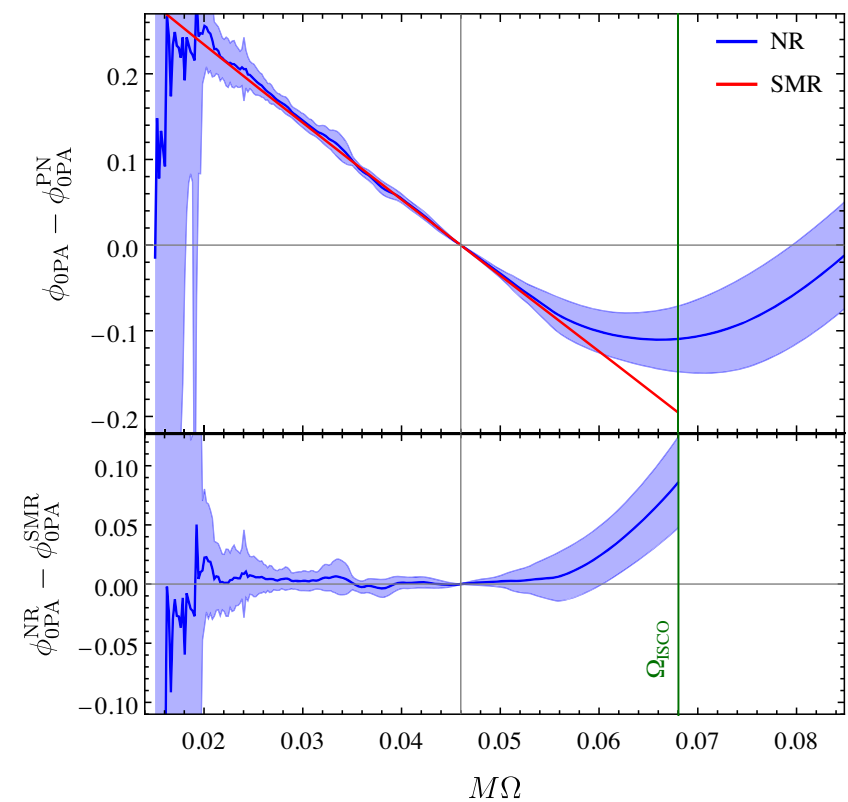

FIG. 1. Top: leading-order in mass-ratio contribution to the orbital phasing of the quasicircular inspiral of nonspinning black holes. Shown are the results derived here from NR simulations, as well as the SMR perturbation theory. For both curves, the 3.5PN result [14] was subtracted for clarity of plotting. Bottom: difference between SMR and the NR result. The shaded areas indicate the estimated uncertainty of the numerical calculation and $\Omega_{\text {ISCO }}$ indicates the last stable orbit for $\nu=0$.

$M \Omega=0.046$. At a given value of $M \Omega$, the postadiabatic coefficients $\phi_{n \mathrm{PA}}(M \Omega)$ are determined by fitting a polynomial in $\nu$ to the data points $\left(\nu_{A}, \phi_{A}^{\mathrm{NR}}(M \Omega)\right)$, where $A=1, \ldots, 55$ labels the NR simulations, and $\nu_{A}$ is the symmetric mass ratio of each simulation. This fit is repeated for many values of $M \Omega$. Error estimates are obtained by repeating this procedure with (i) medium-resolution NR simulations, (ii) using the Weyl scalar $\Psi_{4}$ instead of the GW strain in Eq. (1), (iii) varying the order with which the GW strain is extrapolated to future null infinity, and (iv) changing the number of terms in the fit of form Eq. (3) between three and four. The range of these calculations is reported as error bars in our results. At each frequency, only those NR simulations are used that have a starting frequency below; for $M \Omega \lesssim 0.02$, the reduced number of available NR simulations causes larger error bars.

Results.-The leading-order term $\phi_{\mathrm{OPA}}(M \Omega)$ can be extracted with good accuracy from the NR simulations, as shown in Fig. 1. To reduce the dynamic range on the $y$ axis, this figure shows the difference to the post-Newtonian $\phi_{0 \mathrm{PA}}^{\mathrm{PN}}$ result at order $(v / c)^{7}$, taken from [14]. The blue curve represents the result of our analysis of NR simulations (with error bar), whereas the red line is the leading-order SMR result computed by Eq. (4). The agreement between the two is quite remarkable and is a first indication that the PA expansion of the phase in the mass ratio is well behaved for comparable mass ratios.

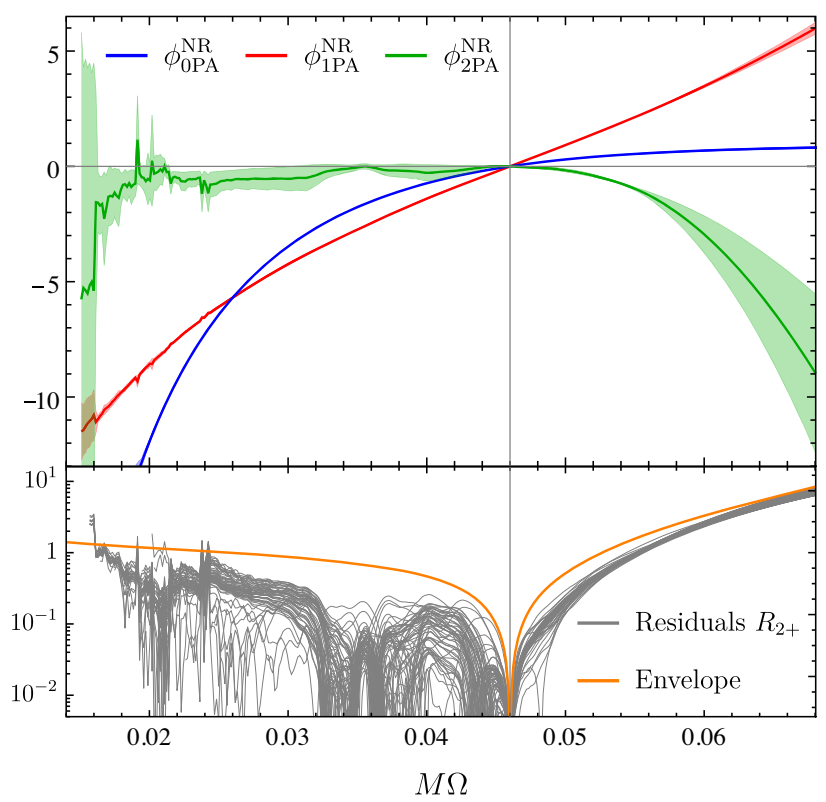

FIG. 2. Top: the three leading terms in the mass-ratio expansion of the orbital phase, as computed here. Bottom: residuals $R_{2+}$ for all $55 \mathrm{NR}$ simulations indicating the combined contributions of 2PA and higher, as well as an envelope bounding these residuals in a $\nu$-independent manner.

At higher frequencies, $M \Omega \gtrsim 0.055$, we find an apparently systematic deviation between NR and the SMR result. This deviation may arise from a breakdown of the PA expansion near the last stable orbit as the binary transitions from inspiral to plunge. Studies of this transition regime $[70,71]$ lead to order $\nu^{-1 / 5}$ corrections to Eq. (3). Including such a term in our fit does indeed eliminate the systematic deviation at $M \Omega \gtrsim 0.055$. However, the additional term is nearly degenerate with the $0 \mathrm{PA}$ and $1 \mathrm{PA}$ terms at low frequencies, making it impossible to get robust numerical results for $\phi_{1 \mathrm{PA}}$ and higher. Therefore, we proceed in our analysis without such transition terms.

Given how well the numerically extracted OPA term agrees with its SMR prediction, we henceforth set it to the SMR value when fitting for the higher-order PA terms. Figure 2 shows the 1PA and 2PA terms obtained from the NR simulations, together with the OPA term already discussed in Fig. 1 . The coefficients $\phi_{n \mathrm{PA}}$ are of comparable magnitude in the frequency range covered by our analysis, suggesting that the PA series is convergent at equal masses. Moreover, for frequencies $M \Omega \lesssim 0.05$, the $2 \mathrm{PA}$ coefficient is almost consistent with zero, i.e., the 0PA and 1PA terms already capture essentially all variation due to mass ratio in the numerical data at these frequencies. In fact, "goodnessof-fit" indicators, such as the adjusted $R^{2}$ value, show only marginal improvements when adding terms to the fit beyond the 1PA coefficient.

The lower panel of Fig. 2 provides a different view on the importance of terms beyond $\phi_{1 \mathrm{PA}}$ : For each of the $55 \mathrm{NR}$ simulations, this panel plots 


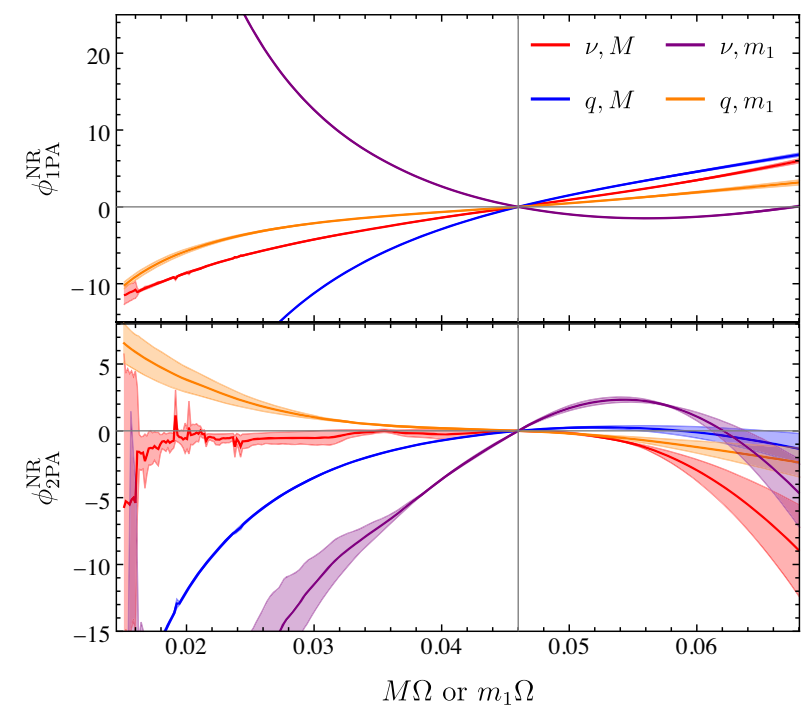

FIG. 3. Impact of the choice of expansion parameters on the 1PA and 2PA contributions to the orbital phasing. The different curves differ in whether in Eq. (3) is expanded in powers of $\nu$ or in $q$ and whether the $\phi$ functions are written in terms of $M \Omega$ or $m_{1} \Omega$. The combination $\nu, M \Omega$ yields an exceptionally small 2PA term at low frequencies.

$$
R_{2+} \equiv \frac{1}{\nu}\left(\phi^{\mathrm{NR}}-\frac{1}{\nu} \phi_{0 \mathrm{PA}}-\phi_{1 \mathrm{PA}}^{\mathrm{NR}}\right)
$$

i.e., the contribution of all terms $n \geq 2$ in Eq. (3), with overall $\nu$ scaling compensated. All $R_{2+}$ can be bounded independent of mass ratio by an envelope function, consisting of the known 3.5 PN terms of $\phi_{2 \mathrm{PA}}$ and a higherorder polynomial in $M \Omega$ fitted by eye.

So far, we have expanded in symmetric mass ratio $\nu$, while scaling orbital frequencies by total mass $M$ [cf. Eq. (3)]. One can also use the mass ratio $q=$ $m_{2} / m_{1}$ as the small parameter and/or scale orbital frequency by the large body's mass $m_{1}$. This yields four variations, all of which agree at the leading OPA order. Figure 3 presents the results for the 1PA and 2PA contributions. In all four cases, the extracted 1PA and 2PA coefficients remain of similar magnitude, implying that the expansion is not dominated by higher-order terms. However, the 2PA term is remarkably small only when expanding using the symmetric mass ratio $\nu$ and total mass $M$. The choice $\nu, M$ is indeed preferred as it is invariant under exchange of the two bodies $1 \leftrightarrow 2$ [20,72].

Discussion.-The phasing of inspiraling $\mathrm{BH}$ binaries is of utmost importance for GW astronomy to find signals, determine their parameters, and perform tests of general relativity. Binaries at intermediate mass ratios $q \sim 10^{-3}$ are in a regime not accessible to NR, while potentially out of reach for SMR perturbation theory. This situation is compounded by the difficulty of calculations of the SMR expansion, for which today only the leading order (called the zeroth postadiabatic order) is fully known. Here,

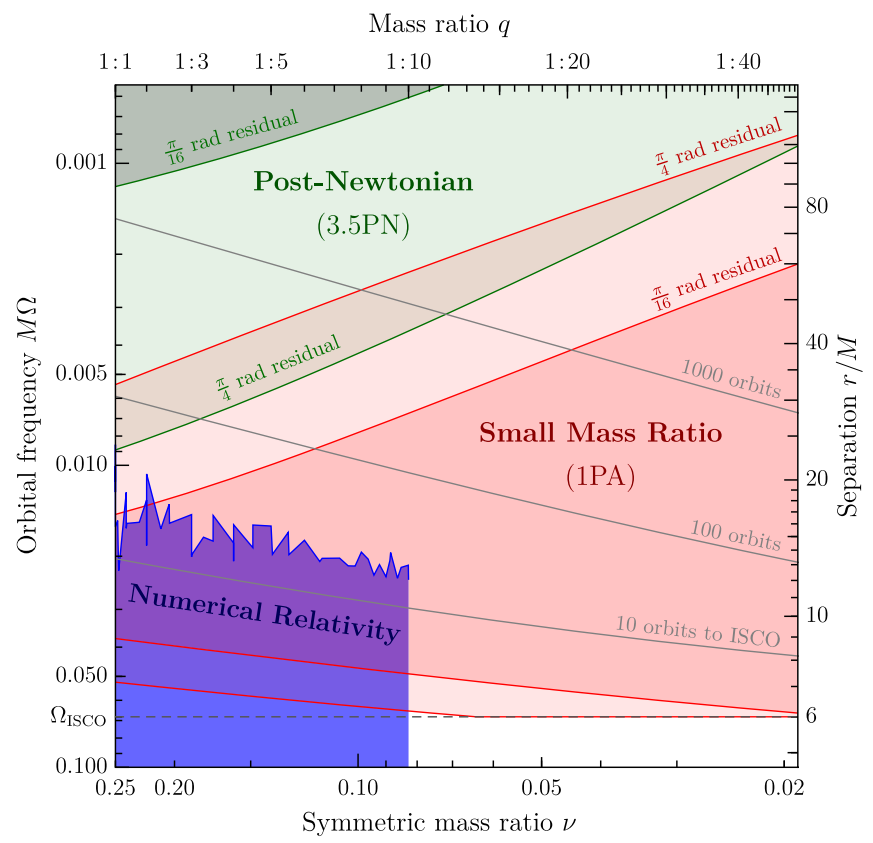

FIG. 4. Region of applicability of different approximation techniques for nonspinning quasicircular binary black hole inspiral. The shaded regions indicate ranges within which the cumulative orbital phase error is less than $\pi / 4$ and $\pi / 16 \mathrm{rad}$, respectively.

we extract the first three terms of the SMR expansion from NR simulations at comparable masses, $q \geq 0.1$, and use these results to perform the first comparison between NR and SMR expanded results for a gauge invariant quantity that includes both dissipative and conservative effects, namely, the accumulated orbital phase as a function of orbital frequency $\phi(M \Omega)$. We have successfully extracted the postadiabatic expansion of this quantity as a power series in the mass ratio from nonspinning quasicircular NR simulations.

The leading adiabatic (OPA) term agrees with the result from SMR calculations. In addition, we obtain a robust determination of the 1PA term, serving as a concrete prediction for the ongoing SMR calculation of this term, which requires the dissipative part of the second-order gravitational self-force. We also estimate the $2 \mathrm{PA}$ term $\phi_{2 \mathrm{PA}}$ from the NR data. Its amplitude is comparable to $\phi_{0 \mathrm{PA}}$ and $\phi_{1 \mathrm{PA}}$ for the frequency range considered here, indicating that the PA expansion remains well behaved. In particular, when the PA series is expanded in powers of the symmetric mass ratio while keeping the total mass fixed, the 2PA and higher-order terms are consistent with zero within the numerical accuracy for $0.015 \lesssim M \Omega \lesssim 0.05$. For higher frequencies (approaching the last stable circular orbit), we find indications of a transition regime to plunge where the series in integer powers of $\nu$ is no longer applicable.

Our analysis allows us to delineate the regions of applicability of SMR, NR, and PN in a quantitative way, as shown in Fig. 4: Assuming $\phi_{1 \mathrm{PA}}$ will become available 
through GSF calculations, the envelope to the $R_{2+}$ in Fig. 2 gives a bound on the secular contributions of higher PA terms. The red shaded areas in Fig. 4 show the largest $M \Omega$ interval that can be covered, such that the total accumulated phase error due to $\geq 2 \mathrm{PA}$ terms is below a certain value. The region of applicability of SMR increases toward smaller mass ratios, but is still non-negligible even at comparable masses. The post-Newtonian errors are estimated by fits against $\phi^{\mathrm{NR}}(M \Omega)$ (cf. top panel of Fig. 1). The green shaded areas indicate regions where the cumulative 3.5 PN phase error for the entire inspiral up to the given frequency is below a certain value. Finally, the blue shaded area indicates the region covered by the NR simulations used here. These simulations have phase accuracy better than the $\pi / 16$ contour line, indicating that the usability of NR is not limited by accuracy but rather by the length of the simulations. The three modeling approaches deliver complementary information, covering different regions of the parameter space. The region of validity of each method depends on the desired accuracy, and it also depends on the use of the waveforms: For GW astronomy, only the accuracy within the frequency band of the relevant GW detectors is important, and this will depend on the total mass of the binary. Moreover, the needed accuracy will depend on the signal-to-noise ratio at which it is observed.

We note that the adiabatic $\phi_{0 \mathrm{PA}}$ term is never accurate enough in the metric of Fig. 4 , because $\phi_{1 \mathrm{PA}}$ contributes tens of radians in the frequency range considered, independent of the mass ratio. This underlines the importance of calculating the 1PA term (and therefore the second-order gravitational self-force) for modeling binaries of any mass ratio. Furthermore, the application of the 1PA approximation for low frequencies is limited by a $(M \Omega)^{-1 / 3}$ divergence of the 2PA term. This motivates the development of models that incorporate both SMR and PN results, e.g., using effective one-body theory [73-76].

The results in this Letter come with two important caveats. First, our results are limited to nonspinning quasicircular black hole binaries. Adding spin or eccentricity makes the waveform considerably more complex and could make the convergence of the PA series significantly worse. Future studies are needed to explore the full parameter space. Even for nonspinning quasicircular case, NR simulations at smaller mass ratio are needed to investigate the transition to plunge, as well as longer simulations, to extend our analysis to smaller frequencies.

Second, the current analysis applies only to the inspiral, since the PA expansion is known to breakdown at the last stable orbit. Our results motivate the development of 1PA accurate models that also include plunge, merger, and ringdown, as has previously been done at OPA order [77].

The authors acknowledge use of public NR data from the SXS Collaboration [27]. *mmeent@aei.mpg.de

[1] J. Aasi et al. (LIGO Scientific Collaboration), Advanced LIGO, Classical Quantum Gravity 32, 074001 (2015); arXiv:1411.4547.

[2] F. Acernese et al. (VIRGO Collaboration), Advanced Virgo: A second-generation interferometric gravitational wave detector, Classical Quantum Gravity 32, 024001 (2015); arXiv:1408.3978.

[3] The GWIC 3G Science Case Team Consortium, The nextgeneration global gravitational-wave observatory: New astrophysics with the farthest, oldest, and most violent events in the Universe (2019), https://gwic.ligo.org/ 3Gsubcomm/documents/science-case.pdf.

[4] P. Amaro-Seoane et al. (LISA Collaboration), Laser interferometer space antenna, arXiv:1702.00786.

[5] B. P. Abbott et al. (LIGO Scientific and Virgo Collaborations), GWTC-1: A Gravitational-Wave Transient Catalog of Compact Binary Mergers Observed by LIGO and Virgo during the First and Second Observing Runs, Phys. Rev. X 9, 031040 (2019); arXiv:1811.12907.

[6] B. P. Abbott et al. (LIGO Scientific and Virgo Collaborations), Binary black hole population properties inferred from the first and second observing runs of Advanced LIGO and Advanced Virgo, Astrophys. J. 882, L24 (2019); arXiv:1811.12940.

[7] T. Venumadhav, B. Zackay, J. Roulet, L. Dai, and M. Zaldarriaga, New binary black hole mergers in the second observing run of Advanced LIGO and Advanced Virgo, Phys. Rev. D 101, 083030 (2020); arXiv:1904.07214.

[8] R. Abbott et al. (LIGO Scientific and Virgo Collaborations), GW190412: Observation of a binary-black-hole coalescence with asymmetric masses, Phys. Rev. D 102, 043015 (2020); arXiv:2004.08342.

[9] R. Abbott et al. (LIGO Scientific and Virgo Collaborations), GW190814: Gravitational waves from the coalescence of a 23 solar mass black hole with a 2.6 solar mass compact object, Astrophys. J. 896, L44 (2020); arXiv:2006.12611.

[10] B. Abbott et al. (KAGRA, LIGO Scientific, and VIRGO Collaborations), Prospects for observing and localizing gravitational-wave transients with Advanced LIGO, Advanced Virgo, and KAGRA, Living Rev. Relativity 21, 3 (2018); arXiv:1304.0670.

[11] K. Jani, D. Shoemaker, and C. Cutler, Detectability of intermediate-mass black holes in multiband gravitational wave astronomy, Nat. Astron. 4, 260 (2020); arXiv:1908 .04985 .

[12] J. Salcido, R. G. Bower, T. Theuns, S. McAlpine, M. Schaller, R. A. Crain, J. Schaye, and J. Regan, Music from the heavens-gravitational waves from supermassive black hole mergers in the EAGLE simulations, Mon. Not. R. Astron. Soc. 463, 870 (2016); arXiv:1601.06156.

[13] M. Volonteri et al., Black hole mergers from dwarf to massive galaxies with the New Horizon and Horizon-AGN simulations, arXiv:2005.04902.

[14] L. Blanchet, Gravitational radiation from post-Newtonian sources and inspiralling compact binaries, Living Rev. Relativity 17, 2 (2014); arXiv:1310.1528.

[15] T. W. Baumgarte and S. L. Shapiro, Numerical Relativity: Solving Einstein's Equations on the Computer (Cambridge University Press, Cambridge, England, 2010). 
[16] E. Poisson, A. Pound, and I. Vega, The motion of point particles in curved spacetime, Living Rev. Relativity 14, 7 (2011); arXiv:1102.0529.

[17] A. Buonanno and T. Damour, Effective one-body approach to general relativistic two-body dynamics, Phys. Rev. D 59, 084006 (1999); arXiv:gr-qc/9811091.

[18] T. Damour, Gravitational scattering, post-Minkowskian approximation and effective one-body theory, Phys. Rev. D 94, 104015 (2016); arXiv:1609.00354.

[19] L. Barack and A. Pound, Self-force and radiation reaction in general relativity, Rep. Prog. Phys. 82, 016904 (2019); arXiv:1805.10385.

[20] A. Le Tiec, A. H. Mroué, L. Barack, A. Buonanno, H. P. Pfeiffer, N. Sago, and A. Taracchini, Periastron Advance in Black Hole Binaries, Phys. Rev. Lett. 107, 141101 (2011); arXiv:1106.3278.

[21] U. Sperhake, V. Cardoso, C. D. Ott, E. Schnetter, and H. Witek, Extreme black hole simulations: Collisions of unequal mass black holes and the point particle limit, Phys. Rev. D 84, 084038 (2011); arXiv:1105.5391.

[22] A. Le Tiec, E. Barausse, and A. Buonanno, Gravitational Self-Force Correction to the Binding Energy of Compact Binary Systems, Phys. Rev. Lett. 108, 131103 (2012); arXiv:1111.5609.

[23] A. Nagar, Gravitational recoil in nonspinning black hole binaries: The span of test-mass results, Phys. Rev. D 88, 121501(R) (2013); arXiv:1306.6299.

[24] A. Le Tiec, A. Buonanno, A. H. Mroué, H. P. Pfeiffer, D. A. Hemberger, G. Lovelace, L. E. Kidder, M. A. Scheel, B. Szilágyi, N. W. Taylor, and S. A. Teukolsky, Periastron advance in spinning black hole binaries: Gravitational self-force from numerical relativity, Phys. Rev. D 88, 124027 (2013); arXiv:1309.0541.

[25] A. Le Tiec and P. Grandclément, Horizon Surface Gravity in Corotating Black Hole Binaries, Classical Quantum Gravity 35, 144002 (2018); arXiv:1710.03673.

[26] M. van de Meent, Self-Force Corrections to the Periapsis Advance around a Spinning Black Hole, Phys. Rev. Lett. 118, 011101 (2017); arXiv:1610.03497.

[27] M. Boyle et al., The SXS Collaboration catalog of binary black hole simulations, Classical Quantum Gravity 36, 195006 (2019); arXiv:1904.04831.

[28] S. Husa, S. Khan, M. Hannam, M. Pürrer, F. Ohme, X. Jiménez Forteza, and A. Bohé, Frequency-domain gravitational waves from nonprecessing black-hole binaries. I. New numerical waveforms and anatomy of the signal, Phys. Rev. D 93, 044006 (2016); arXiv:1508.07250.

[29] C. O. Lousto and J. Healy, Exploring the Small Mass Ratio Binary Black Hole Merger Via Zeno's Dichotomy Approach, arXiv:2006.04818 [Phys. Rev. Lett. (to be published)].

[30] A. H. Mroue et al., Catalog of 174 Binary Black Hole Simulations for Gravitational Wave Astronomy, Phys. Rev. Lett. 111, 241104 (2013); arXiv:1304.6077.

[31] T. Hinderer and E. E. Flanagan, Two timescale analysis of extreme mass ratio inspirals in Kerr. I. Orbital Motion, Phys. Rev. D 78, 064028 (2008).arXiv:0805.3337.

[32] M. van de Meent and N. Warburton, Fast Self-forced Inspirals, Classical Quantum Gravity 35, 144003 (2018); arXiv:1802.05281.
[33] Black Hole Perturbation Toolkit, bhptoolkit.org.

[34] R. Fujita and H. Tagoshi, New numerical methods to evaluate homogeneous solutions of the Teukolsky equation, Prog. Theor. Phys. 112, 415 (2004); arXiv:gr-qc/0410018.

[35] R. Fujita, W. Hikida, and H. Tagoshi, An efficient numerical method for computing gravitational waves induced by a particle moving on eccentric inclined orbits around a Kerr black hole, Prog. Theor. Phys. 121, 843 (2009); arXiv:0904 .3810 .

[36] W. Throwe, High precision calculation of generic extreme mass ratio inspirals, 2010, MIT Bachelor's thesis.

[37] M. van de Meent, Resonantly enhanced kicks from equatorial small mass-ratio inspirals, Phys. Rev. D 90, 044027 (2014); arXiv:1406.2594.

[38] M. van de Meent and A. G. Shah, Metric perturbations produced by eccentric equatorial orbits around a Kerr black hole, Phys. Rev. D 92, 064025 (2015); arXiv:1506.04755.

[39] M. van de Meent, Gravitational self-force on eccentric equatorial orbits around a Kerr black hole, Phys. Rev. D 94, 044034 (2016); arXiv:1606.06297.

[40] M. van de Meent, Gravitational self-force on generic bound geodesics in Kerr spacetime, Phys. Rev. D 97, 104033 (2018); arXiv:1711.09607.

[41] L. Barack and N. Sago, Gravitational self force on a particle in circular orbit around a Schwarzschild black hole, Phys. Rev. D 75, 064021 (2007); arXiv:gr-qc/0701069.

[42] L. Barack and N. Sago, Gravitational self-force on a particle in eccentric orbit around a Schwarzschild black hole, Phys. Rev. D 81, 084021 (2010); arXiv:1002.2386.

[43] S. Akcay, N. Warburton, and L. Barack, Frequency-domain algorithm for the Lorenz-gauge gravitational self-force, Phys. Rev. D 88, 104009 (2013); arXiv:1308.5223.

[44] T. Osburn, E. Forseth, C. R. Evans, and S. Hopper, Lorenz gauge gravitational self-force calculations of eccentric binaries using a frequency domain procedure, Phys. Rev. D 90, 104031 (2014); arXiv:1409.4419.

[45] C. Merlin and A. G. Shah, Self-force from reconstructed metric perturbations: Numerical implementation in Schwarzschild spacetime, Phys. Rev. D 91, 024005 (2015); arXiv:1410.2998.

[46] A. Pound, Second-Order Gravitational Self-Force, Phys. Rev. Lett. 109, 051101 (2012); arXiv:1201.5089.

[47] A. Pound, Nonlinear gravitational self-force. I. Field outside a small body, Phys. Rev. D 86, 084019 (2012); arXiv:1206 6538 .

[48] A. Pound and J. Miller, Practical, covariant puncture for second-order self-force calculations, Phys. Rev. D 89, 104020 (2014); arXiv:1403.1843.

[49] A. Pound, Conservative effect of the second-order gravitational self-force on quasicircular orbits in Schwarzschild spacetime, Phys. Rev. D 90, 084039 (2014); arXiv:1404.1543.

[50] A. Pound, Second-order perturbation theory: Problems on large scales, Phys. Rev. D 92, 104047 (2015; arXiv:1510 .05172 .

[51] J. Miller, B. Wardell, and A. Pound, Second-order perturbation theory: The problem of infinite mode coupling, Phys. Rev. D 94, 104018 (2016); arXiv:1608.06783.

[52] A. Pound, Nonlinear gravitational self-force: Second-order equation of motion, Phys. Rev. D 95, 104056 (2017); arXiv:1703.02836. 
[53] A. Pound, B. Wardell, N. Warburton, and J. Miller, SecondOrder Self-Force Calculation of the Gravitational Binding Energy in Compact Binaries, Phys. Rev. Lett. 124, 021101 (2020); arXiv:1908.07419.

[54] G. B. Cook and H. P. Pfeiffer, Excision boundary conditions for black hole initial data, Phys. Rev. D 70, 104016 (2004); arXiv:gr-qc/0407078.

[55] M. Caudill, G. B. Cook, J. D. Grigsby, and H. P. Pfeiffer, Circular orbits and spin in black-hole initial data, Phys. Rev. D 74, 064011 (2006); arXiv:gr-qc/0605053.

[56] G. B. Cook and B. F. Whiting, Approximate killing vectors on $S^{* * 2}$, Phys. Rev. D 76, 041501(R) (2007); arXiv:0706 .0199 .

[57] G. Lovelace, R. Owen, H. P. Pfeiffer, and T. Chu, Binaryblack-hole initial data with nearly-extremal spins, Phys. Rev. D 78, 084017 (2008); arXiv:0805.4192.

[58] H. P. Pfeiffer, D. A. Brown, L. E. Kidder, L. Lindblom, G. Lovelace, and M. A. Scheel, Reducing orbital eccentricity in binary black hole simulations, Classical Quantum Gravity 24, S59 (2007); arXiv:gr-qc/0702106.

[59] L. T. Buchman, H. P. Pfeiffer, and J. M. Bardeen, Black hole initial data on hyperboloidal slices, Phys. Rev. D 80, 084024 (2009); arXiv:0907.3163.

[60] H. Friedrich, On the hyperbolicity of Einstein's and other gauge field equations, Commun. Math. Phys. 100, 525 (1985).

[61] D. Garfinkle, Harmonic coordinate method for simulating generic singularities, Phys. Rev. D 65, 044029 (2002); arXiv:gr-qc/0110013.

[62] F. Pretorius, Numerical relativity using a generalized harmonic decomposition, Classical Quantum Gravity 22, 425 (2005); arXiv:gr-qc/0407110.

[63] L. Lindblom, M. A. Scheel, L. E. Kidder, R. Owen, and O. Rinne, A new generalized harmonic evolution system, Classical Quantum Gravity 23, S447 (2006); arXiv:gr-qc/ 0512093.

[64] O. Rinne, Stable radiation-controlling boundary conditions for the generalized harmonic Einstein equations, Classical Quantum Gravity 23, 6275 (2006); arXiv:gr-qc/0606053.

[65] O. Rinne, L. Lindblom, and M. A. Scheel, Testing outer boundary treatments for the Einstein equations, Classical Quantum Gravity 24, 4053 (2007); arXiv:0704.0782.
[66] M. A. Scheel, H. P. Pfeiffer, L. Lindblom, L. E. Kidder, O. Rinne, and S. A. Teukolsky, Solving Einstein's equations with dual coordinate frames, Phys. Rev. D 74, 104006 (2006); arXiv:gr-qc/0607056.

[67] M. Boyle and A. H. Mroue, Extrapolating gravitationalwave data from numerical simulations, Phys. Rev. D 80, 124045 (2009); arXiv:0905.3177.

[68] C. J. Woodford, M. Boyle, and H. P. Pfeiffer, Compact binary waveform center-of-mass corrections, Phys. Rev. D 100, 124010 (2019); arXiv:1904.04842.

[69] SXS Gravitational Waveform Database, https://data.blackholes.org/waveforms/.

[70] A. Buonanno and T. Damour, Transition from inspiral to plunge in binary black hole coalescences, Phys. Rev. D 62, 064015 (2000); arXiv:gr-qc/0001013.

[71] A. Ori and K.S. Thorne, The transition from inspiral to plunge for a compact body in a circular equatorial orbit around a massive, spinning black hole, Phys. Rev. D 62, 124022 (2000); arXiv:gr-qc/0003032.

[72] A. Le Tiec, The overlap of numerical relativity, perturbation theory and post-Newtonian theory in the binary black hole problem, Int. J. Mod. Phys. D 23, 1430022 (2014); arXiv: 1408.5505.

[73] A. Antonelli, M. van de Meent, A. Buonanno, J. Steinhoff, and J. Vines, Quasicircular inspirals and plunges from nonspinning effective-one-body Hamiltonians with gravitational self-force information, Phys. Rev. D 101, 024024 (2020); arXiv:1907.11597.

[74] T. Damour, Gravitational self force in a Schwarzschild background and the effective one body formalism, Phys. Rev. D 81, 024017 (2010); arXiv:0910.5533.

[75] S. Akcay, L. Barack, T. Damour, and N. Sago, Gravitational self-force and the effective-one-body formalism between the innermost stable circular orbit and the light ring, Phys. Rev. D 86, 104041 (2012); arXiv:1209.0964.

[76] S. Akcay and M. van de Meent, Numerical computation of the EOB potential $q$ using self-force results, Phys. Rev. D 93, 064063 (2016); arXiv:1512.03392.

[77] N. E. M. Rifat, S. E. Field, G. Khanna, and V. Varma, Surrogate model for gravitational wave signals from comparable and large-mass-ratio black hole binaries, Phys. Rev. D 101, 081502(R) (2020); arXiv:1910.10473. 